\title{
Approche husserlienne et approche linguistique du signe
}

Alain Flajoliet

\section{OpenEdition}

1 Journals

Édition électronique

URL : http://journals.openedition.org/alter/1377

DOI : $10.4000 /$ alter. 1377

ISSN : 2558-7927

Éditeur :

Association ALTER, Archives Husserl (CNRS-UMR 8547)

\section{Édition imprimée}

Date de publication : 1 octobre 2011

Pagination : 83-106

ISBN : 978-2-9522374-7-5

ISSN : $1249-8947$

\section{Référence électronique}

Alain Flajoliet, " Approche husserlienne et approche linguistique du signe », Alter [En ligne], 19 | 2011, mis en ligne le 01 janvier 2020, consulté le 23 janvier 2020. URL : http://journals.openedition.org/alter/ 1377 ; DOI : 10.4000/alter.1377 


\title{
APPROCHE HUSSERLIENNE ET APPROCHE LINGUISTIQUE DU SIGNE
}

\author{
Alain Flajoliet
}

Cette étude essaie de dégager certaines convergences et différences entre l'approche husserlienne de la conscience de signe dans les Recherches logiques au tournant du siècle (l'édition de 1913 ne modifie pas fondamentalement les descriptions acquises en 1901) et certains courants linguistiques issus de la révolution saussurienne dans l'analyse du langage. Pour l'essentiel nous limiterons nos références d'une part aux travaux que Benveniste, Jakobson, Martinet ont consacré au problème de l'articulation du sens à la forme phonique et d'autre part a la refondation de la sémantique par Greimas et Hjelmslev. Cette confrontation n'ignorera pas les oppositions très manifestes entre une description dont le centre reste la visée de sens subjective qui porte la constitution des significations des objets (ainsi que les relations cohérentes et réglées entre ces significations) et une approche où les considérations «sémantiques » tendent à se subordonner à l'analyse " sémiotique » de la forme du signe comme suite de phonèmes ${ }^{1}$. Nous empruntons à Benveniste cette distinction entre élucidation sémantique et sémiotique du signe, qui nous servira de fil conducteur².

\footnotetext{
${ }^{1}$. Les considérations sémiotiques ou sémantiques ne sont pas exclusives l'une de l'autre comme on le voit par exemple en phonologie, mais l'une peut prédominer sur l'autre. Cf. les deux articles bien connus de Benveniste qui ont constitué un point d'appui essentiel de l'analyse du langage par Ricœur lors de son tournant linguistique des années soixante. En principe l'élucidation de tel ou tel niveau du langage ne peut être purement sémiotique purement sémantique, les deux démarches s'appuient l'une sur l'autre. Toutefois la phrase fait exception, elle ne relève que d'une analyse sémantique. Benveniste, Problèmes de linguistique générale t. 1, Paris, Gallimard, 1966, p. 119-131 : «Les niveaux de l'analyse linguistique ». T. 2, Paris, Gallimard, 1974, p. 215-240 : «La forme et le sens dans l'analyse linguistique ». Abrév. PLG 1, 2. Ricœur, La métaphore vive, Paris, Seuil, 1975, p. 88-99.

${ }^{2}$. En limitant la discussion touchant aux rapports entre linguistique et phénoménologie à la seule description husserlienne du signe, donc en excluant la conception husserlienne de la syntaxe (cf. Recherche IV, l'analyse de la grammaire pure), notre exposé est fondamentalement incomplet. Nous lèverons cette abstraction dans une étude ultérieure.
} 
Notre première partie situera le terrain de la confrontation.

Notre seconde partie comparera la manière dont la phénoménologie et la linguistique pensent l'articulation du sens et du son. Nous nous appuierons sur le concept de "niveaux d'analyse » distincts du langage proposé par Benveniste. Les deux principaux sont celui du signe (du mot) et celui de la phrase (de l'énoncé). La phrase possède un statut spécifique parce qu'elle constitue le niveau le plus élevé, elle ne se subordonne à aucun niveau supérieur. Elle ne $\mathrm{s}^{\prime}$ « intègre », dans la terminologie de Benveniste, à aucune unité linguistique supérieure. Le niveau du signe, lui, se subordonne à celui de la phrase, ou si l'on préfère l'unité de signification $\mathrm{s}^{\prime}$ « intègre » à la phrase. La relation doit être lue aussi et surtout de haut en bas : la phrase intègre en elle les mots. Le signe ou mot n'est évidemment pas l'unité linguistique ultime, il est l'unité d'un sens et d'une "forme » (sonore, graphique). À son tour la forme sonore peut être analysée soit en tant qu'elle s'intègre au sens - les considérations sémantiques restent alors essentielles -, soit en restant au même niveau - elle s'analyse alors en composants (traits distinctifs) à l'aide d'une méthode distributionnelle typiquement sémiotique. Sous son premier aspect, la phonologie (par exemple celle du Cercle de Prague) conserve un aspect sémantique puisque les phonèmes ne sont pas seulement dégagés comme des entités oppositives et négatives au sein du système phonologique mais aussi comme possédant la fonction de discriminer les unités significatives. Mais sous son second aspect elle ouvre la voie à une analyse plus profonde, $\mathrm{d}^{\prime}$ ordre purement sémiotique, où chaque phonème réalise une combinaison des mêmes traits distinctifs sonores élémentaire (les phèmes) différente de celle réalisée par tous les autres phonèmes du système. Ces remarques révèlent déjà les convergences et les divergences entre la phonologie et la phénoménologie husserlienne du signe. Dans l'analyse des rapports entre le son et le sens de l'expression, la description husserlienne accentue la relation de haut en bas : l'énoncé idéal intègre en lui les unités de significations idéales qui intègrent en elles les sons euxmêmes idéalisés. La linguistique à l'inverse, tout en reconnaissant la valeur de la relation du son au sens pour la connaissance des phonèmes, promeut du point de vue ontologique la relation de bas en haut: des traits distinctifs différentiels acoustiques aux phonèmes et des phonèmes au sens.

On peut développer (ce sera l'objet de notre troisième partie) la confrontation entre linguistique et phénoménologie husserlienne sur un autre terrain, celui de l'analyse de la signification dans ses rapports à l'objet auquel elle se rapporte. Husserl défend dans la Recherche I l'existence pour toute signification d'un rapport nécessaire à l'objet qu'elle signifie. Mais cela ne va plus de soi pour la linguistique contemporaine. Chez Hjelmslev et Greimas, toute signifi- 
cation est justiciable d'une analyse en pure immanence s'inspirant de celle qui a réussi pour l'analyse des phonèmes en traits distinctifs acoustiques purement oppositifs. Toute signification est justiciable d'une analyse distributionnelle en pure immanence qui la résout dans une combinatoire ordonnée de sèmes dont chacun n'est que sa différence d'avec tous les autres.

\section{Le terrain phénoménologique de l'analyse du signe}

1.1 Husserl, l'unité idéale de la signification et la dépsychologisation de l'analyse du signe

Acte, contenu, objet constituent les trois éléments fondamentaux de la conscience de signification pour Husserl en 1901. Très schématiquement, on dira que l'acte constituant une conscience de signe appréhende des contenus sensoriels (auditifs acoustiques et kinesthésiques) pour former un contenu de signification par lequel un objet est signifié ${ }^{3}$. On doit ajouter pour être complet l'existence d'un acte « remplissant» la signification qui donne, au lieu de l'objet «luimême » (nécessairement donné), ce même objet «en chair et en os » qui, lui, peut n'être pas donné lorsque la signification est comprise. Pour cette raison, nous négligerons dans cette étude l'acte remplissant.

Une première remarque s'impose. La confrontation avec la linguistique que nous commençons ici pourrait s'arrêter immédiatement si nous prenions comme point d'appui ces travaux qui dans les années soixante avaient voulu par souci de rigueur scientifique éliminer de l'analyse linguistique toute référence au sens et au sujet. Nous nous autorisons à négliger cette orientation, perceptible par exemple chez Bloomfield ${ }^{4}$, parce qu'elle a été marginalisée par la plupart des travaux récents, de Chomsky à Benveniste en passant par Jakobson, Hjelmselv et Greimas.

Chez Saussure comme chez Jakobson, Hjelmslev et Benveniste, la description de la signification du signe - c'est-à-dire du « signifié », du «sens», du «plan du contenu»-, ne se résorbe nullement dans la description de la face physique de ce même signe - c'est-à-dire de l'aspect « signifiant », « phonologique », ou encore de ce qui relève du «plan de l'expression» ou de la «forme»-, tout en étant uni

\footnotetext{
3. Conformément à la volonté explicite de Husserl en 1901, nous ne distinguons pas «signification » et «sens».

4. Le sens («meaning ») d'un mot chez Bloomfield tend - en simplifiant beaucoup - à se réduire à son emploi au sein d'une communauté linguistique, chacun devenant tout à tour locuteur pour d'autres auditeurs et auditeur pour d'autres locuteurs, l'apprentissage s'effectuant par les progrès d'une communication comprise selon le modèle stimulus-réponse.
} 
intimement à la face physique ${ }^{5}$. Si en outre on tient compte du fait que la linguistique, au moins en première analyse, s'est progressivement attachée à donner (de Benveniste à Courtès) un statut au sujet du discours ou de l'énonciation ${ }^{6}$, nous pouvons dire, à vue de pays, qu'il n'existe pas d'obstacle de principe à une confrontation entre certains aspects de la linguistique du signe et la phénoménologie du signe dans la Recherche I.

Avant de rentrer de façon plus précise dans l'interprétation des analyses husserliennes de la conscience de signification en les confrontant à celle des linguistes, il est nécessaire de préciser sur quel terrain ces analyses se situent. Nous les envisageons sauf mention explicite sans les retouches, d'ailleurs minimes, apportées par la seconde édition. Ce terrain, celui d'une " phénoménologie » de l'expression, n'est pas immédiatement disponible puisqu'on ne peut y accéder qu'en réduisant ce qui ne relève pas essentiellement du phénomène expressif. L'indice, comme renvoi d'une réalité à une autre, totalement pris dans la naturalité, est réduit en premier lieu parce que le renvoi qui le constitue n'est aucunement porté par une visée consciente de signification, mais par des associations habituelles produisant la conviction que "si le premier, alors le second ». Ainsi commence le processus par lequel phénomène expressif est arraché à la psychologie empirique, au fil conducteur (révélateur) de la manifestation de l'irréductible écart entre une démonstration authentique, fondée sur des lois idéales de déductibilité, progressant de propositions évidentes en propositions évidentes, et les renvois successifs motivés des vécus psychologiques dans lesquels les propositions sont données ${ }^{7}$. Au contraire, les renvois empiriques sont soumis à la juridiction des lois idéales de déductibilité. Mais ce n'est pas tout: le processus de réduction du psychologico-factuel se poursuit avec $l^{\prime}$ élimination $\mathrm{du}$ "discours communicatif ${ }^{8}$. Car dans cette fonction le discours, cessant de signifier pour manifester les vécus du locuteur, est totalement ressaisi par l'empirico-pychologique. Le phénomène de l'expression n'apparaît donc que dans la « vie psychique solitaire » où l'on nomme des objets par leurs significations hors de tout contexte de communication à autrui ${ }^{9}$.

\footnotetext{
${ }^{5}$. La distinction proprement husserlienne passe entre la «face physique» (le «complexe phonique ») et la « signification» (le "sens»). Cf. Logische Untersuchungen, zweiter Band, Husserliana Band XIX/1, La Haye, M. Nijhoff, 1984, p. 38-39 et 43-45, Abrév. LU XIX/1 ; trad. fr. Recherches logiques, Paris, P.U.F., 1969, t. II, 1, p. 36-37 et 42-44, Abrév. RL II/1.

${ }^{6}$. J. Courtès, Analyse sémiotique du discours, Paris, Hachette, 1991, p. 143-152. Abrév. ASD. Benveniste, $P L G, 1$, Partie V ; PLG, 2, Partie V.

7. LU XIX/1 p. 32 sq. ; RL II/, p. 30 sq. L'écart n'empêche pas les motivations psychologiques de $\mathrm{s}^{\prime}$ inscrire sous les lois idéales de déductibilité.

8. $L U X \mathrm{XIX} / 1$ p. 39 sq. ; RL II/1, p. 37 sq.

${ }^{9}$. $L U$ XIX/1, p. $41 ; R L I I / 1$, p. 40 . Pour sa part, Jakobson, à la suite de K. Bühler (« Axiomatik der Sprachwissenschaft », Kant-Studien, no 38, Berlin, 1933, p. 19-90), conteste qu' on puisse exclure de
} 
La réduction ainsi effectuée ne nous place pas encore au niveau proprement phénoménologique des distinctions idéales. L'analyse commence en effet par déployer une description de l'expression (donc dans la vie psychique solitaire) qui reste encore au niveau de la psychologie descriptive. Husserl dégage d'abord (\$ 9-10) les « distinctions phénoménologiques » (en fait psychologico-descriptives) issues de la réduction (acte donateur de sens, contenu sonore animé, relation à l'objet) et passe seulement ensuite - dans une sorte de seconde réduction - aux «distinctions idéales» (\$11-12) dont la description commence explicitement au paragraphe $11^{10}$. Le premier mouvement descriptif reste encore au niveau d'une élucidation du côté subjectif et psychologique de l'expression, mais c'est pour se hausser ensuite au ton des distinctions idéales authentiquement phénoménologiques. L'analyse, prise dans son mouvement d'ensemble de dépassement de la description psychologique vers le concept d'unité idéale de la signification, pourrait s'interpréter comme représentant le mouvement même de constitution de la psychologie eidétique de l'expression dans son équivoque structurelle. On devrait souligner alors que l'essence de l'expressivité ne réside pas dans quelques topos ouranios, elle est l'essence de cet acte psychologique, comme aussi d'une infinité d'autres en lesquelles elle peut se réaliser ; inversement, que l'acte d'expression décrit psychologiquement ne saurait être abandonné à sa contingence, il s'inscrit sous la loi de l'essence idéale de l'expressivité. Toutefois, on doit remarquer que le terrain conquis pour la description proprement phénomenologique (dépsychologisée) de l'expression est celui de l'idéal et non de l'essence, ce qui introduit une nuance non négligeable. L'idéal n'est pas exactement l'essence, même si l'essence est une idéalité. Il ne possède pas comme l'essence de rapport à une infinité de cas factuels possibles, et en ce sens il apparaît délié de ce rapport au factuel qui reste fondamental dans la pensée de l'essence. Conséquemment, interpréter phénoménologiquement l'expression comme unité idéale, $c^{\prime}$ est subir l'attraction d'une forme de logicisation de la grammaire. Et corrélativement, la description psychologique de la conscience expressive persiste comme ce dans quoi se réalise l'unité idéale de la signification.

\footnotetext{
l'analyse du langage la fonction de communication. Cf. Essais de linguistique générale, t. 1, Paris, Editions de Minuit, 1963, p. 213 sq., Abrév. EPLG, 1. Comme on le sait depuis les analyses classiques de Derrida, le risque de cette réduction du psychologique et de la sphère de la communication est d'aboutir à une forme de logicisation du langage, réduit à n'être que la manifestation transparente d'une pure pensée idéale se réfléchissant elle-même. Cependant l'Introduction au tome 2 des Recherches affirme que la grammaire ne peut se résorber simplement dans la logique (en dépit d'un parallélisme entre les deux disciplines).

${ }^{10}$. Les numéros des paragraphes renvoient au chapitre 1 de la Recherche I. Les citations aux passages : $L U X I X / 1$, p. 43 ; RL II/1, p. 42 ; $L U X I X / 1$, p. 48 ; RL II/1, p. 48.
} 
La facilité avec laquelle la description de la conscience expressive est dépsychologisée et haussée au ton des distinctions idéales de la phénoménologie eidétique dès 1901 (puisque ces pages n'ont pas eu à subir de retouches essentielles) mérite attention. Elle contraste avec la persistance dans l'ensemble des Recherches d'un grand nombre d'analyses de pure psychologie descriptive qui ont dû être remaniées voire supprimées en 1913. La raison en est que la conscience de signification a ceci d'absolument spécifique qu'elle ne peut pas être décrite de manière satisfaisante au niveau simplement psychologico-descriptif qui per definitionem exclut toute prise en compte de ce qui est constitutif d'une conscience de signe: la visée idéale de sens. Cela, Husserl l'avait compris dès 1896. En effet, il objectait à Twardowski dans une "Discussion » ${ }^{11}$ inédite, que s'il était légitime de distinguer psychologiquement l'acte, le contenu et l'objet d'une représentation, il n'était nullement légitime de transposer purement et simplement cette distinction psychologique à l'analyse de la signification. Car on ne saurait, disait-il, identifier la signification d'un nom désignant un objet avec le contenu de la représentation correspondante de l'objet. L'analyse de la conscience de signification ne peut être que " fonctionnelle» (Husserl dira plus tard : « idéale »). Le seul aspect de la conscience expressive qui demeurait de nature psychologique en 1896 était donc le datum sonore que la conscience animait et qui était présent réellement (au sens de : reell) dans l'immanence des vécus. Mais il était considéré comme inessentiel par rapport à la visée significative en tant qu'identité «d'une multiplicité illimitée d'actes individuellement et réellement séparés ${ }^{12}$ qui, elle, constituait l'essence de la conscience expressive. En 1901, Husserl affirme que même le datum sonore psychologique peut subir l'idéalisation, de sorte que c'est la totalité de la description de l'expression qui peut être effectuée sur le plan de l'idée ${ }^{13}$. Ce plan, précise-t-il, est celui où les distinctions deviennent pleinement objectives : «Le point de vue subjectif fait place au point de vue objectif ${ }^{14}$.

La première conséquence de cette démarche qui reconduit la description psychologique à la phénoménologiques des aspects idéaux de la signification, c'est que les distinctions psychologiques produites en premier lieu subissent l'attraction des distinctions idéales auxquelles elles sont déjà promises au moment même où on les établit. On devine par exemple que les distinctions effectuées au niveau psychologique entre le phénomène physique de l'expression, l'acte

\footnotetext{
11. «Discussion » de Twardowski, inédit tiré de Hua Band XXII, Aufsätze und Rezensionen (18901910), trad. fr. J. English, in Husserl-Twardowski, Sur les objets intentionnels, Paris, Vrin, 1993, p. 349-356, Abrév. SOI.

12. SOI, p. 350.

13. $L U$ XIX/1, p. $48-49 ; R L$ II/1, p. 49.

14. $L U X \mathrm{XIX} / 1$, p. $48 ; R L \mathrm{II} / 1$, p. 49.
} 
donateur de sens et le rapport de la signification à l'objet sont destinées à être reversées au compte des distinctions idéales. D'autres indices de cette attraction sont perceptibles. Le concept psychologique de «fusionnement » issu de Stumpf ${ }^{15}$, utilisé dans la Recherche I (au paragraphe 3 du chapitre 1) pour décrire l'unité entre les trois composantes essentielles de l'expression (son, signification, visée d'objet), réapparaît dans la Recherche III au sein de la théorie des rapports entre objets indépendants et dépendants (chapitre 1, § 8-9), donc dans le cadre d'une élucidation eidétique et a priori. Notons aussi que Husserl s'autorise à fixer au sein même des premières analyses psychologiques ce qui définit "essentiellement » l'expres$\operatorname{sion}^{16}$.

La seconde conséquence est que le rapprochement entre la phénoménologie de l'expression et la linguistique du signe s'inscrira toujours dans certaines limites. Dépsychologiser comme l'entreprend Husserl la conception du signe en l'inscrivant dans une pensée de l'idéalité (signification idéale, son idéal, rapport idéal de la signification l'objet) ne peut pas être totalement assimilé à cette dépsychologisation de l'interpretation du signe à laquelle a procedé par exemple le Cercle de Prague pour construire la phonologie. Les travaux de ce Cercle s'inscrivent au sein d'un projet d'analyse scientifique possédant un contenu empirique. Même chez un linguiste comme Hjelmslev, qui dans ses Prolégomènes à une théorie du langage construit une véritable théorie linguistique de type axiomatisé (fondée sur un système de définitions susceptibles d'engendrer par déduction les différentes propositions de la théorie), cette derniere relève encore de la connaissance empirique (et non de la philosophie) parce qu'une fois construite (par voie non inductive) elle doit $\mathrm{s}^{\prime}$ appliquer à $\mathrm{l}^{\prime}$ expérience ${ }^{17}$. La théorie hjelmslevienne peut bien prétendre a un degré très élevé d'abstraction elle n'est pas comme chez Husserl une description idéalisée du langage ${ }^{18}$. La science linguistique contemporaine est parvenue à dégager des concepts généraux - et même des généralités de niveau très élevé (des " catégories ») -, mais ce ne sont pas des idéalités parce qu'elles n'ont de sens que par leur applicabilité aux langues empiriques. Par exemple, comme

\footnotetext{
15. Stumpf, Tonpsychologie, Leipzig, 1885 et 1890, § 16 .

16. C'est l'acte conférant la signification qui représente l'essence de l'expression ( $L U X \mathrm{XIX} / 1, \mathrm{p} .43$; $R L \mathrm{II} / 1, \mathrm{p} .43)$.

17. Hjelmslev, Prolégomènes à une théorie du langage, Paris, Editions de Minuit, 1966, p. 19, 20-22, 23-25. Abrév. PTL.

${ }^{18}$. La "grammaire générative» de Chomsky s'établit sur la base d'une situation de parole idéalisée. Cf. Aspects de la théorie syntaxique, trad. fr. J.-Cl. Milner, Paris, Seuil, 1957, p. 42 : «Une grammaire peut être considérée comme la théorie d'une langue; elle est descriptivement adéquate pour autant qu'elle décrit correctement la compétence intrinsèque du sujet indigène idéal ». On voit cependant que le renvoi à la « compétence » du sujet maintient la grammaire dans l'orbe des théories empiriques.
} 
l'expliquent Greimas et Courtès, les catégories grammaticales (c'est-àdire les concepts fondamentaux de toute grammaire, autant dire les concepts les plus généraux sur lesquels reposent la morphologie et la syntaxe ${ }^{19}$, sont définies par la linguistique comme des généralités conceptuelles abstraites mais pas comme des idéalités. On voit en effet que la définition linguistique des catégories, à la différence de la définition phénoménologique, ne contient aucune référence au domaine des distinctions idéales. Ce sont « des objets grammaticaux $\mathrm{qu}^{\prime}$ on désigne aussi comme classes d'ordre paradigmatique (obtenus par substitution dans la chaîne syntagmatique d'unités de type choisi). On aura ainsi a) des classes "morphologiques" ou "parties du discours" (substantif, adjectif, verbe, etc.) ; b) des classes "syntaxiques" ou fonctionnelles (sujet, objet, prédicat, épithète, etc.) ; c) des classes "syntagmatiques" ou syntagmes (verbal, nominal) $»^{20}$. La différence avec les catégories grammaticales qui chez Husserl soutiennent les règles de la grammaire pure, est patent : ces dernières fondent des lois a priori idéales de la signification (non pas formelles, il s'agit d'un a priori matériel).

On a souvent insisté sur l'inadéquation de l'orientation phénoménologique qui tend à déporter toute la description de l'expression vers l'élucidation de distinctions idéales, ${ }^{21}$ mais peut-être serait-il juste de remarquer aussi qu'en rendant problématique la validité d'une pure description psychologique de l'expression, elle a favorisé certains rapprochements avec la linguistique, notamment avec la linguistique qui émergeait à Prague dans le sillage du Cours de linguistique générale de Saussure. En se dégageant de l'emprise de la psychologie, la phénoménologie de la Recherche I prenait ses distances avec nombre de travaux qui, par des voies variées que nous ne pouvons étudier ici, avaient subordonné l'étude du langage à la psychologie, et elle retrouvait certaines positions critiques des Pragois 22 .

\footnotetext{
19. Greimas, Courtès, Dictionnaire raisonné de la théorie du langage (abrév. DRTL) article «catégorie».

20. Greimas, Courtès, DRTL, article « catégorie ».

21. Merleau-Ponty, Les sciences de l'homme et la phénoménologie, Cours de la Sorbonne, Paris, Centre de Documentation Universitaire, 1975, p. 52-61 ; Derrida, "La forme et le vouloir dire ", Marges de la philosophie, Paris, Editions de Minuit, 1972, p. 187-207.

22. Wundt, Völker Psychologie, I, Die Sprache, 1800. H. Paul, Prinzipien der Sprachgeschichte, $1^{\text {re éd. }}$ 1886, rééd. Darmstadt, Wissenschaftlische Buchgesellschaft, 1960. L'histoire des langues pour H. Paul se fonde sur la psychologie parce que «l'élément psychique est le facteur essentiel de tout mouvement de la culture » (op. cit., p. 6). A. Marty, Untersuchungen zur Grundlegung der allgemeinen Grammatik und Logik, Band I, Halle, M. Niemeyer, 1908. Élève de Brentano, Marty réactive comme Husserl le projet d'une grammaire générale, sur un terrain qui reste assez largement celui d'une éidétique descriptive. (Son maître ouvrage sur la grammaire subit toutefois l'influence de Humboldt via la distinction entre « forme intérieure » et « forme extérieure » du langage).
} 

signe

\section{2 Le cercle de Prague, l'étude fonctionnelle et non psychologique du}

Le mouvement de dépsychologisation de l'analyse du signe que nous avons observé dans la phénoménologie se retrouve mutatis mutandis dans les travaux du Cercle de Prague. Dans le sillage de Saussure, mais de manière beaucoup plus ferme que ce dernier, la linguistique du Cercle a conquis son terrain dans le premier quart du siècle en libérant la linguistique du poids de l'approche purement phonétique qui dominait soit dans l'approche des «néogrammairiens ", soit dans les recherches centrées sur l'étude psycho-acoustique et motrice du langage ${ }^{23}$. Jakobson montre dans ses Six leçons sur le son et le sens que la phonétique, quoique représentant une importante discipline progressant constamment dans la connaissance des mouvements articulatoires et des sons impliqués dans les langues, ne constitue que le porche de la linguistique, faisant même obstacle à cette dernière dès lors qu'elle interdit dogmatiquement toute étude des rapports entre le son et le sens ${ }^{24}$. Toute explication causale et naturaliste est strictement subordonnée à la description de phénomènes signifiants. Cette position nous rapproche incontestablement de démarche phénoménologique des Recherches logiques en 1901.

Jakobson a reconnu lui-même cette proximité théorique en rappelant qu'entre le Cercle de Prague et le mouvement phénoménologique les contacts furent nombreux et positifs ${ }^{25}$. Déjà le Cercle linguistique de Moscou, préfiguration de celui de Prague, fondé par Jakobson et quelques autres linguistes en 1915, accueillit Gustav Špet, élève de Husserl, ce qui fut l'occasion de discussions sur l'application linguistique possible du concept de grammaire pure des Recherches

\footnotetext{
23. Jakobson, Six leçons sur le son et le sens (abrév. SLSS), Paris, Minuit, 1976, ch. 1. Les néogrammairiens, qui dominent la linguistique allemande de la seconde moitié du $19^{\mathrm{e}}$ siècle, se regroupent autour d'un domaine privilégié d'étude (l'indo-européen comme racine d'une multitude de langues) et d'une méthodologie: il faut étudier les sons des langues en les comparant les unes aux autres dans la simultanéité et selon l'évolution historique, dans le but de dégager des lois phonétiques strictes (B. Malmberg, Histoire de la linguistique, Paris, P.U.F., 1991, ch. 11. Abrév. HL.

24. En effet (Jakobson, SLSS, p. 29), dans une approche purement phonétique du son (ramené in fine à des mouvements articulatoires), il devient quasi impossible d'identifier chaque son au sein d'une continuité de processus.

25. ELG, 2, p. 12-13. La proximité ici soulignée n'empêche aucunement l'existence de différences importantes sur d'autres points essentiels. Le concept de "phonème » par exemple élaboré au sein du Cercle de Prague, n'est pas assimilable à celui de son idéal proposé par Husserl dans son analyse de la face physique du mot. En outre, l'infléchissement toujours plus marqué de la phénoménologie vers une philosophie transcendantale l'a éloignée d'une discipline linguistique que Jakobson a toujours conçue comme science empirique utilisant le formalisme mathématique. Sur les liens entre le mouvement phénoménologique et les Pragois, cf. E. Holenstein, R. Jakobsons Phänomenologischer Strukturalismus, Frankfurt, Suhrkampf, 1975, en partic. p. 13-15, 18-19, 22-25, 39-40, Abrév. JPS.
} 
logiques ${ }^{26}$. Plusieurs élèves de Husserl ont travaillé activement au sein du Cercle linguistique de Prague (Landgrebe, Pos, Čiževskij). Husserl lui-même prononça en 1935 devant le Cercle, sur l'initiative de Jakobson, une conférence sur la phénoménologie du langage ${ }^{27}$.

\section{Structure générale de l'expression}

Rappelons ce qui est acquis: l'analyse husserlienne du signe, d'abord psychologique et descriptive, est redéployée ensuite au niveau proprement phénoménologique des distinctions idéales. Entrons dans l'analyse du signe et commençons par préciser que l'expression élémentaire est comprise par Husserl aussi bien comme "signe » que comme "mot». Plus rigoureuse que cette approche (conforme à la linguistique traditionnelle), la linguistique contemporaine s'essaye à distinguer le mot et le signe et surtout à ne conserver que le second concept $\mathrm{t}^{28}$.

Les premières analyses phénoménologiques au sens d'une psychologie descriptive peuvent se résumer ainsi. Il faut distinguer en premier lieu entre ce qui est essentiel à l'expression - un «acte conférant la signification » (ou « intention de signification ») et venant " animer » un « complexe phonique », pour constituer la « référence » à un objet -, et ce qui s'adjoint éventuellement à ce premier acte : $l^{\prime}$ « acte remplissant la signification », réalisant la donation de l'objet signifié, et le manifestant en chair et en os $^{29}$. En outre est affirmée l'unité par fusionnement des trois aspects constituant ce qu'il y a d'essentiel de l'expression ${ }^{30}$. Redéployons cette description psychologique au niveau de la structure idéale de l'expression - d'empirique et subjective la description devient idéale et objective ${ }^{31}$. Une expression a pour aspect essentiel d'avoir une signification idéale (sur la

\footnotetext{
26. Jakobson, ELG, 2, p. 12. Cf. B. Malmberg, HL, p. 417-418).

27. Jakobson, ELG, 2, p. 13, Holenstein, JPS, p. 12.

28. Courtès et Greimas montrent (DRTL, article «Mot») comment on peut essayer de faire l'économie du mot en lui substituant le concept de «lexie » (B. Pottier), ou mieux de « lexème » (Greimas), ou encore en construisant (Chomsky) la syntaxe phrastique non à partir de classes morphologiques mais à partir de classes syntagmatiques (syntagme nominal, verbal, etc.). Cependant aucune de ces tentatives n'est totalement convaincante, ce qui justifie la conservation de la notion de «mot» en linguistique et, rétrospectivement la conceptualité traditionnelle (qui est celle de Husserl).

29. $L U X \mathrm{XX} / 1$, p. $44-45 ; R L \mathrm{II} / 1$, p. $43-44$.

30. $L U X \mathrm{XIX} / 1$, p. $45 ; R L \mathrm{II} / 1$, p. 45. (Trad fr. modifiée). Et titre du $\S 10$ («L'unité phénoménologique de ces actes »). «Fusionnement » ne signifie pas que les trois aspects constitueraient ne masse confuse et inarticulée (cf. A. Gurwitsch, Théorie du champ de conscience, Paris, 1957, Desclée de Brouwer, p. 72-77).

31. LU XIX/1, p. 48 ; RL II/1, p. 49. Cette affirmation ne vaut en fait que lorsque l'objet signifié est lui-même un objet idéal (comme en logique ou en mathématique) car alors il est effectivement délié des actes qui le donnent.
} 
base d'un datum acoustique ou graphique idéalisé) et, par cette signification, de constituer un rapport idéal à son objet. Je distingue, dit Husserl ${ }^{32}$ :

a) La signification dans sa pureté idéale, dégagée par réflexion idéalisante sur les vécus psychologiques concrets passagers et multiples, signification que toute expression possède nécessairement. On pourrait définir la signification ainsi comprise : l'unité idéale du signifier, distinguée des multiples actes psychiques que cette unité subsume. Un exemple emprunté aux mathématiques illustre cette définition. Soit l'expression " les trois hauteurs d'un triangle se coupent en un seul point ». Les individus proférant cette phrase manifestent des vécus variables au fil du temps et multiples selon leurs dispositions psychiques. Mais cette multiplicité fluente se subsume sous l'unité idéale fixe du signifier se rapportant dans son idéale présence à luimême à l'état de choses mathématiques idéal en intégrant un concretum sonore lui-même idéalisé33. L'unité idéale de la signification est la composante absolument essentielle de l'expression dans la perspective d'une élucidation phénoménologique idéale. Une expression ne possédant pas de signification mais seulement une forme phonique n'est donc qu'une pseudo expression. Il peut s'agir d'une pure forme phonique (Abracadabra) ou de plusieurs expressions dont les significations ne s'accordent pas pour former une signification unitaire (Vert est ou).

b) La signification comme unité idéale s'appuie sur une perception de la face physique du signe elle-même idéalisée.

c) La signification contient un rapport nécessaire idéal à un objet signifié. Ce rapport pose plusieurs difficultés sur lesquelles nous reviendrons lors de la confrontation au concept linguistique de référence.

d) L'intuition remplissante ; elle s'ajoute à l'expression en venant en recouvrement avec elle pour former un acte de connaissance ${ }^{34}$. Si les deux actes se « recouvrent », la signification se remplit, elle acquiert

\footnotetext{
32. $L U X \mathrm{XX} / 1$, p. 59-60; RL II/1, p. 61-62.

${ }^{33}$. Il ne s'agit pas d'une phrase banale, mais de l'expression d'un théorème mathématique. La conception husserlienne de la signification prend ses appuis aussi bien dans des exemples empruntés au langues naturelles - «le vainqueur d'Iéna », « un merle s'envole », « Londres », « il y a des gâteaux »-, qu'au langage servant à exprimer des vérités logiques et mathématiques. Il ne va évidemment pas de soi que la conception du signe et de la signification se dégageant de l'étude des langues naturelles doive être la même que celle se dégageant d'une étude du «langage » logico-mathématique». Cette difficulté n'est autre que celle, plus générale, liée au projet d'une "grammaire pure ». C'est bien une grammaire, nécessairement liée aux contingences des langues particulières ; mais c'est une grammaire pure, dont les règles sont des règles idéales (de cohérence entre les significations). Voir notre Conclusion.
}

34. $L U X \mathrm{XIX} / 1$, p. $61-62 ; R L \mathrm{II} / 1$, p. 64. 
une «plénitude» («Fülle»), selon le concept que Husserl réinvestira dans la sixième Recherche ${ }^{35}$.

Nous pouvons déjà esquisser quelques remarques concernant la manière dont la linguistique ayant assumé l'héritage saussurien se situe par rapport à cette conception husserlienne du signe. Pour reprendre une distinction faite dans notre Introduction, on pourrait dire que dans la phénoménologie husserlienne du signe, la relation de haut en bas est privilégiée parce que c'est la visée idéale de signification en suspens dans l'énoncé lui-même idéal qui constitue l'essence de l'expression et que c'est à elle que tout s'ordonne, la suite de sons idéalisée. Dans l'approche des linguistiques post saussuriens, même si la phonologie prolonge ces considérations en définissant les phonèmes dans leur fonction de discrimination du sens, il reste que $\mathrm{c}^{\prime}$ est inversement la relation de bas en haut qui tend à dominer ontologiquement: le système des traits distinctifs acoustiques oppositifs constitue les phonèmes et ceux-ci à leur tour supportent les unités de signification.

\section{Le son et le sens}

\subsection{Husserl et la prééminence de la relation intégrative}

Nous avons vu, en dégageant au niveau de la psychologie de l'expression l'unité, foncière de l'acte porteur de la signification et de la perception du datum sonore qu'il anime. Une précision décisive s'impose maintenant: dans cette unité, il y a "non équivalence ", " inegalité de valeur» («Ungleichwertigkeit») des composantes respectives $»^{36}$ de l'acte expressif. Autrement dit, la perception $d u$ mot comme forme sonore (ou graphique) se subordonne à la visée de signification. Cela ne va pas de soi puisqu'en linguistique ce déséquilibre en faveur de la signification n'existe pas. L'argument de Husserl repose sur l'évidence de la perception interne (ce qui en affaiblit la portée du point de vue d'une description eidétique rigoureuse mais aussi du point de vue d'une linguistique ayant surmonté tout psychologisme). Le sujet parlant est censé pouvoir vérifier par perception interne que dans la compréhension du sens d'un mot proféré, il vit dans la conscience de la signification (et de l'objet), nullement dans celle de la forme sonore du mot $^{37}$. Seule la premiere est au premier plan dans le

\footnotetext{
35. $L U X \mathrm{XIX} / 1$, p. $50 ; R L \mathrm{II} / 1, \mathrm{p} .51$.

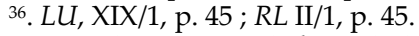

${ }^{37}$. Dans la conceptualité psychologique on dira que la « représentation du mot » n'est jamais ce dans quoi la conscience du sujet vit primairement. «L'une et l'autre, la représentation du mot et l'acte donateur de sens sont vécus ; mais, tandis que nous vivons la représentation du mot, nous ne vivons cependant pas le moins du monde dans l'acte de nous représenter le mot, mais
} 
champ de conscience, la seconde est totalement à l'arrière plan. (Psychologiquement d'ailleurs, rien ne serait plus difficile que de renverser ce rapport entre avant plan et premier plan, ou de l'annuler). L'integration de la perception du datum sonore à l'intention de signification modifie essentiellement cette perception, qui acquiert précisément une fonction expressive qu'elle ne possédait pas auparavant.

Le passage aux distinctions idéales maintient l'inégalité aperçue au niveau psychologique. La perception fugitive du datum sonore s'effaçant au profit de la forme sonore idéalisée et l'acte psychologique variable conférant la signification devenant l'unité idéale du signifier, $l^{\prime}$ '«inégalité de valeur» désormais passe entre cette forme sonore objective-idéale et la signification objective-idéale à laquelle elle se subordonne complètement. Non seulement cette intégration vaut du point de vue de la connaissance, mais elle tend à valoir aussi du point de vue de l'être parce que Husserl ne conçoit pas la forme phonique comme un authentique fondement de l'unité idéale de la signification. C'est ce qui fait principalement problème pour l'approche linguistique. En effet cette dernière, tout en admettant que sans la signification la forme phonologique $n^{\prime}$ aurait aucune fonction (ce qui implique que la forme ne peut être connue qu'à partir de son intégration dans la signification), n'oublie jamais que la signification dépend ontologiquement de la forme phonologique qui pour sa part se constitue dans son autonomie au sein du systeme phonologique. La forme phonologique devient un authentique support de la signification, ce que Husserl refuse explicitement dans la Recherche V38. Il explique qu'un acte complexe peut être formé de deux actes plus simples dont l'un est fondé sur l'autre, mais que ce n'est aucunement nécessaire et que ce second cas est précisément celui de l'expression. Admettons que l'acte donateur de la face physique de l'expression et l'acte donateur de la signification soient comme deux actes simples dont se compose l'acte expressif total. L'acte donateur de la face physique de l'expression ne fonde pas l'acte donateur de la signification. En revanche en logique, dans la prédication catégorique, l'acte total s'articule en deux actes partiels plus simples, celui qui donne le sujet et celui qui donne le prédicat, mais ici la position du sujet sert bien de base à la position du prédicat qui est attribué.

Il est donc tout à fait cohérent que dans ce même passage de la Recherche V Husserl réaffirme avec force la stricte subordination de la

\footnotetext{
exclusivement dans l'accomplissement de son sens, dans l'acte de signifier $(L U, \mathrm{XIX} / 1, \mathrm{p} .46 ; R L$ II/1, p. 45). Lorsque Husserl parle d'un acte de signification qui «trouve son appui (seine Stütze findet) » dans le contenu intuitif de la représentation du $\operatorname{mot}(L U \mathrm{XIX} / 1$, p. 47 ; RL II/1, p. 47), il confirme le rôle subordonné de la perception du datum sonore par rapport à la compréhension du sens.
}

38. $L U$ XIX/1, p. 419-422; RL II/2, p. 211-214. 
forme sonore au sens. Il va même jusqu'à affirmer que le complexe phonique est «inessentiel » par rapport à la signification et qu'il pourrait «disparaître complètement $»^{39}$. Formule qu'il ne faut pas prendre au sens littéral, Husserl veut simplement dire que le complexe phonique pourrait être remplacé par n'importe quel autre de manière arbitraire, sans que la signification en soit altérée. Toutefois, la conséquence de cette thèse demeure bien l'inégalité foncière entre la forme sonore et l'unité idéale de la signification, ce qui entraîne fort loin des positions de la linguistique. Certes Saussure affirmait bien $l^{\prime}$ «arbitraire»du signifiant par rapport au signifié mais, outre le fait que cette thèse était établie dans une perspective psychologique ${ }^{40}$, elle était rectifiée par d'autres passages du Cours ${ }^{41}$ et elle ne conduisait pas du tout à l'inégalité posée par Husserl ${ }^{42}$. Du coup, le signifiant recevait un pouvoir de donner forme au signifié, de contribuer à l'articulation du niveau du sens, affirmation que l'on retrouve chez Jakobson, Benveniste, Martinet, mais que la phénoménologie husserlienne du signe exclut. Dans cette dernière la forme sonore, malgré son unité avec la signification, conserve une certaine extériorité par rapport à elle : n'importe quelle forme sonore pourrait faire l'affaire pour exprimer une signification donnée. La signification ne porte donc pas véritablement la marque de la forme sonore, qui en retour ne contribue pas véritablement à la constituer. Sur ce point les orientations de la linguistique vont dans la direction exactement opposée. Même s'il ne s'agit jamais (au moins chez les linguistes mentionnés) de réduire le sens à la forme sonore, il s'agit bien toujours de privilégier l'analyse "de bas en haut ». Dans l'ordre du connaître, on concédera que la forme s'intègre au sens et que l'analyse de la forme phonologique n'est possible qu'en intégrant cette dernière à la signification. Mais dans une perspective ontologique tout se renverse et c'est la forme qui porte le sens et contribue à le constituer. Chez Husserl en revanche la tendance est à l'inverse de ne pas faire dépendre du

\footnotetext{
39. $L U X \mathrm{XIX} / 1$, p. $421 ; R L \mathrm{II} / 2$, p. 213.

40. Elle revient à poser que le choix d'un signifiant pour un signifié est immotivé.

41. Dans son commentaire où il rectifie en 1939 la formule du Cours touchant au soi disant arbitraire du rapport entre signifiant et signifié, Benveniste affirme qu'entre signifiant et signifié il existe un rapport nécessaire (c'est seulement, entre l'objet désigné par le signe et le signe que le rapport est arbitraire) ( $P L G, 1$, p. 51) en ajoutant que c'était bien là la véritable doctrine de Saussure

42. Saussure affirme plutôt l'égalité parfaite de deux faces du signe: signifiant et signifié sont exactement sur le même plan, ils sont comme le recto et le verso d'une même feuille de papier (Cours de linguistique générale, Paris, Payot, 1972, p. 145, Abrév. CLG). Et : signifiant et signifié « sont intimement unis et s'appellent l'un l'autre (CLG, p. 99). La langue rapporte la pensée et la substance phonique l'une à l'autre en les articulant simultanément (CLG, p. 156). "Cette combinaison produit une forme et non une substance" (CLG, p. 157). Cette perspective va marquer la phonologie (pas d'analyse possible de la forme phonologique sans le recours aux significations qu'elle informe) et la lexicologie (pas d'analyse possible des significations sans appui sur l'étude des signifiants porteurs des significations).
} 
mot le sens de la forme sonore quant à sa constitution et de ne le considérer que comme intégrant de la phrase. Il affirme en effet la complète indifférence de l'unité idéale de la signification à la forme. L'effacement de la relation de bas en haut dans l'analyse aboutit à une conception paradoxale que l'approche linguistique ne peut admettre (tout en reconnaissant, comme on le voit chez Jakobson, la valeur de la dépsychologisation opérée par la phénoménologie dans l'étude du signe ainsi que de l'interprétation du signe comme essentiellement lié au sens).

Portons maintenant notre attention sur la manière dont la linguistique envisage les rapports entre le son et le sens. Par rapport à Husserl, deux novations majeures apparaissent immédiatement. En premier lieu, la signification n'est pas pensée comme unité idéale, en second lieu, la forme sonore est pensée comme articulation de phonèmes. Ces deux novations contiennent la raison des divergences que nous avons commencé de faire apparaître.

3.2 La dépendance ontologique en linguistique du sens par rapport à la forme phonique

Nous l'avons déjà remarqué à la suite de Jakobson, la phonologie est née en se détachant d'une approche purement phonétique des sons et des processus moteurs d'articulation formant la langue, c'està-dire en considérant ces sons comme porteurs d'une fonction dans la signification des signes ${ }^{43}$. L'analyse phonologique par essence ne perd jamais de vue l'intégration de la forme sonore au sens (ce qui constitue un point de contact avec la phénoménologie). " $\mathrm{Si}$ le phonème se définit, écrit Benveniste, c'est comme constituant d'une unité plus haute, le morphème ${ }^{44}$. Saussure, malgré la regrettable affirmation de l'Introduction de son Cours selon laquelle la phonologie n'est pas différente de la phonétique, avait déjà eu l'intuition juste que la forme sonore qui importe à l'analyse proprement linguistique des mots est constituée non pas de sons pris dans leur substantialité, mais de phonèmes qui s'opposent les uns aux autres au sein d'un système, pour assumer leur fonction de discriminer les unités significatives. Établir la liste des différents phonèmes suppose, comme l'a mis en lumière Benveniste, une méthode qui utilise simultanément la segmentation et la substitution ${ }^{45}$. D'abord il convient de segmenter les unités significatives jusqu'aux plus petites : les mots, ou plus exactement les «morphèmes » de l'analyse en constituants immédiats, ou encore les «monèmes» (Martinet). Il faut

\footnotetext{
43. Cf. aussi A. Martinet, Éléments de linguistique générale, Paris, A. Colin, 1974, p. 61-64.

44. PLG, 1, p. 122.

45. PLG, 1, p. 119-120. C'est aussi la démarche phonologique standard pour Martinet (op. cit., p. 61-100) et Jakobson ( $E L G, 1$, p. 101-157 ; SLSS, ch. IV et V).
} 
ensuite segmenter la forme phonique de ces unités signifiantes, ce qui suppose d'utiliser la méthode de substitution. A ce niveau, nous sommes encore dans une démarche qui préserve une certaine affinité avec la démarche phénoménologique puisque ce sont les unités significatives qui servent de point d'appui et de guide pour l'analyse de la forme, mais en même temps déjà dans une démarche qui n'est plus phénoménologique puisqu'il ne s'agit pas de dégager un datum sonore substantiel, mais une forme sonore composée de phonèmes qui ne sont que des entités différentielles et oppositives ${ }^{46}$.

En effet les segments sonores recherches ne sont pas des data sonores élémentaires mais des entités oppositives dégagées par la méthode de substitution. Pour chaque unité sonore minimale possible, on multiplie les essais de substitution (de commutation) et on observe si, en introduisant telle nouvelle unité sonore minimale, une signification persiste (quoique modifiée) ou non. Si une signification persiste, on dira que l'unité sonore nouvelle fait partie de la classe des substituts possibles de l'unité de départ et que c'est un nouveau phonème. Toute classe de phonèmes commutables est un "paradigme ${ }^{47}$. La convergence avec l'approche phénoménologique réside dans le fait que la forme sonore n'est connue que dans et par son intégration au sens, la différence tient au fait que la définition de la forme sonore chez Husserl demeure la définition d'un datum substantiel idéalisé, alors que dans l'approche linguistique cette définition est purement différentielle et fonctionnelle (mais pas idéale). $\mathrm{Ni}$ chez Benveniste, ni chez Martinet, ni chez Jakobson il n'existe de possibilité de définir le phonème à part et dans sa nature propre et chez aucun d'entre eux il ne possède le statut d'unité idéale (c'est une simple unité fonctionnelle). Insistons sur le statut d'entité purement relative $\mathrm{du}$ phonème. $\mathrm{D}^{\prime}$ abord la définition paradigmatique d'un phonème est une définition purement différentielle: chaque phonème est défini parmi une classe $\mathrm{d}$ 'autres phonèmes substituables, chacun étant capable, s'il est introduit, de produire une nouvelle signification. Si l'on ajoute ensuite que la définition syntagmatique du phonème (par sa relation in praesentia avec d'autres phonèmes qu'il précède ou qu'il suit) est elle aussi différentielle, on voit que le phonème est radicalement étranger à toute forme de substantialité. Il se définit, explique Benveniste, « par l'ensemble des environnements où il se présente, et au moyen d'une double relation, relation du [phonème] avec les autres [phonèmes] simultanément présents dans la même portion de l'énoncé (relation syntagmatique), relation du phonème avec les autres [phonèmes] mutuellement substituables (relation paradigmatique) ${ }^{48}$. Ou, pour le dire de manière synthé-

46. «Les phonèmes sont avant tout des entités relatives, oppositives et négatives », CLG, p. 164.

47. DRTL, article « phonème».

48. PLG I, p. 120 
tique : les phonèmes sont « des classes syntagmatiques obtenues par commutations paradigmatiques $\gg^{49}$.

Toutefois l'opposition de l'approche linguistique à l'approche phénoménologique se radicalise lorsqu'on poursuit l'analyse phonologique à un niveau plus profond que celui exigé par la simple connaissance des phonemes. En admettant que la connaissance et l'identification des différents phonèmes suppose de les envisager essen-tiellement comme s'intégrant au sens, il demeure qu'en sens inverse le sens est matériellement fonction des phonèmes qui le portent et qui se constituent à leur propre niveau "mérismatique » (Benveniste) à travers le jeu des « traits distinctifs » différentiels ou «phèmes ». Or ce niveau ultime est celui de l'inscription corporelle du signe. Jakobson donne l'exemple du système consonantique du français. Il place toutes les consonnes à l'intérieur d'un tableau à double entrée permettant de croiser, pour la détermination de chaque phonème, plusieurs séries de traits distinctifs purement oppositifs qui relèvent: soit du point d'articulation de la voix, soit de l'opposition « nasal » vs « oral » (avec la sous distinction occlusif vs constrictif), soit enfin de l'opposition « centrifuge » (le point d'articulation se situe en arrière du résonateur buccal) vs « centripète » (le point d'articulation se situe en avant du résonateur buccal) $)^{50}$.

À ce niveau terminal de l'analyse du signe, l'intégration du signe au sens a disparu des préoccupations du linguiste, en même temps que l'approche sémantique est totalement passée à l'arrière plan. Le dernier niveau atteint est celui de l'analyse sémiotique (distributionnelle) du phonème en ses composants, qui n'envisage ces derniers que comme des entités différentielles et négatives au sein d'un systeme d'oppositions. Ontologiquement la forme phonique, dans ses traits distinctifs acoustiques et moteurs corporels, apparaît à cette profondeur ultime niveau comme supportant nécessairement le signe. Une série de divergences essentielles apparaît ici entre les perspectives de la phénoménologie et de la linguistique. Divergence entre une analyse qui se place au niveau de l'unité idéale de signification, et une analyse qui réinscrit le signe dans la corporéité. Entre d'une part l'affirmation de l'inégalité entre la visée de sens et le point d'appui constitué par la forme sonore, et d'autre part l'affirmation selon laquelle c'est au sein des différences de la forme sonore que la signification peut se constituer. Entre la thèse selon laquelle la forme sonore ne peut être étudiée que comme intégrant la signification (dans une perspective sémantique), et la thèse selon laquelle la forme sonore relève en dernière instance d'une analyse sémiotique et distributionnelle qui reconduit aux traits distinctifs acoustiques purement

49. DRTL, article «phonologie ».

50. SLSS, p. 97-104. 
différentiels. Manifestement une opposition plus générale traverse toute cette série d'oppositions particulières : celle qui sépare une phénoménologie du signe qui reste centrée sur la visée intentionnelle (idéalisée) du sens et une linguistique qui relativise l'importance de cette visée en la renvoyant en dernière instance à une série d'oppositions de traits distinctifs corporels s'opposant différentiellement au sein d'un système non intentionnel (bien qu'il porte l'intention significative).

\section{Le rapport de la signification à l'objet}

4.1 Husserl : la signification, quoique distincte essentiellement du rapport à l'objet, se rapporte nécessairement à ce dernier

L'approche husserlienne donnant toujours la prééminence à la visée de sens, non seulement le son est primordialement l'intégrant $\mathrm{du}$ mot, mais encore le mot à son tour est primordialement l'intégrant de la phrase (de l'énoncé). Et comme énoncer c'est nécessairement dire quelque chose à propos de quelque chose, le mot du point de vue phénoménologique possède nécessairement un rapport à un objet ${ }^{51}$. C'est sur ce rapport que porte maintenant notre analyse. Dans ce nouveau cadre il faut clarifier le lien que Husserl établit entre la visée idéale de signification analysée précédemment et le rapport à l'objet.

Ce lien est travaillé par une tension. D'une part la signification porte le rapport à l'objet - "dans la signification se constitue le rapport à l'objet $»^{52}-$, mais d'autre part le rapport à l'objet est distinct de la pure visée signification. "Toute expression, non seulement exprime quelque chose, mais elle énonce encore sur quelque chose ; elle n'a pas seulement sa signification, mais elle se rapporte à des objets quels qu'ils soient. [...] Mais jamais l'objet ne coïncide avec la signification $»^{53}$. Husserl précise en outre un point important : il est indifférent que l'objet auquel la signification se rapporte existe ou soit fictif, voire impossible.

Le premier aspect de la thèse de Husserl est qu'il ne faut pas confondre l'unité idéale de la signification comme telle et son rapport à l'objet. "Nous pouvons considérer comme assurée la distinction entre la signification d'une expression et sa propriété de se diriger en le nommant, tantôt vers telle réalité objective, tantôt vers telle autre (et naturellement aussi la distinction entre la signification et l'objet

\footnotetext{
51. Cf. Strawson, Les individus, Paris, Seuil, 1973. En tout énoncé, à la fonction prédicative s'adjoint nécessairement une fonction identifiante.

52. $L U X \mathrm{XIX} / 1$, p. 59 ; RL II/1, p. 61.

53. $L U X \mathrm{XIX} / 1$, p. $53 ; R L I I / 1$, p. 52 . (2e éd.).
} 
lui-même $»^{54}$. Husserl donne deux arguments. 1) Une signification comme unité idéale parfaitement identifiée n'a pas nécessairement le même objet, mais son objet peut varier, tandis qu'inversement un objet parfaitement identifié n'est pas nécessairement donné par la même signification mais par plusieurs significations différentes ${ }^{55}$. 2) Le défaut d'objet n'est nullement identique au défaut de signification. C'est ce que prouve la distinction essentielle fixée dans la Recherche IV entre l'absurdité sémantique ( Unsinn») ou absence de signification et le contresens ontologique («Widersinn») ou absence de rapport à l'objet.

Le second aspect de la thèse husserlienne, c'est d'affirmer qu'entre la signification et l'objet le lien est nécessaire et non pas contingent, de sorte qu'une analyse de la signification en pure immanence est tout simplement impossible. Nommer, c'est nommer quelque chose comme tel ou tel, tout comme énoncer, c'est dire quelque chose à propos de quelque chose. Les Leçons de 1908 réaffirmeront que l'objectité désignée n'est structurée catégorialement par rien d'autre que par l'articulation significative, tandis que symétriquement l'articulation significative n'est rien d'autre que la catégorisation de l'objectité désignée ${ }^{56}$.

Il existe bien une tension entre ces deux aspects. Le second lie étroitement le domaine de désignation et d'articulation des formations de sens au domaine des objets, le premier autonomise le domaine des significations par rapport au domaine des objets. Cette tension est perceptible aussi dans le domaine de la logique formelle tel que le déploie Logique formelle et logique transcendantale. L'apophantique formelle et l'ontologie formelle sont à la fois distinctes et étroitement solidaires. Les catégories de signification (formelles) qui règlent tout le domaine des jugements formalisés en fondant la logique formelle sont indissociables des catégories ontologiques qui règlent les articulations des corrélats objectifs de ces jugements en fondant la mathématique formelle.

Avant de montrer comment un certain courant linguistique illustré par les travaux de Greimas et de Hjelmslev s'oppose à ce type d'analyse par la construction d'une théorie sémantique qui met entre parenthèses le rapport de la signification à l'objet, rappelons que la

\footnotetext{
54. $L U X \mathrm{XIX} / 1$, p. $54 ; R L \mathrm{II} / 1$, p. 55.

55. Le premier cas est un cas d'homonymie : des expressions possédant la même signification, mais se réfèrent à des objets différents. Le second cas est un cas de synonymie.

56. Les formes catégoriales interviennent dès la description du simple nom parce que Husserl ne sépare pas absolument le niveau du mot et celui de la phrase. Le nom est promis à son intégration dans l'énoncé, et l'énoncé peut de son côté subir une nominalisation. Dans la Recherche logique I, des exemples comme "le vainqueur d'Iéna", "le vaincu de Waterloo » peuvent s'analyser comme des énoncés implicites (cf. la théorie des descriptions définies de Russel). Parfois c'est un énoncé prédicatif explicite qui sert à Husserl de base descriptive pour son élucidation d'une signification expressive.
} 
position phénoménologique (Husserl le reconnaît) contient déjà en elle-même de réelles difficultés. Nous laissons de côté le problème posé par le rapport des expressions " essentiellement occasionnelles » a leurs objets, étroitement lié à la conception proprement husserlienne de l'unité idéale de la signification ${ }^{57}$, pour nous attacher à la question des significations sans objet ${ }^{58}$. Cette question ne peut être résolue qu'en évitant deux écueils: la fausse identification de la signification au rapport à l'objet, et l'oubli du nécessaire rapport de toute signification a un objet. Les analyses de Husserl manifestent un certain embarras. Par exemple il commente une formule correcte ( «c'est dans la signification que se constitue le rapport à l'objet ») par une formule douteuse "employer une expression avec sens et se rapporter par une expression à l'objet [...] c'est là une seule et même chose $»^{59}$. Pour clarifier la discussion, il faut sans doute mobiliser la distinction entre «Unsinn » et «Widersinn »: posséder une signification pour une expression relève de la cohérence sémantique tandis que se référer à l'objet relève de la non contradiction de la signification, ce sont donc bien deux choses distinctes. En même temps le lien entre ces deux choses est manifeste : une expression qui serait incohérente sémantiquement («Vert est ou») ne pourrait même pas prétendre se rapporter à un objet tandis qu'inversement une signification cohérente sémantiquement («le vainqueur d'Iéna ») peut légitimement prétendre se rapporter à un objet. La difficulté comme on le voit, $c^{\prime}$ est que cette pretention des expressions sémantiquement cohérentes n'est pas toujours justifiée, comme on le voit avec l'expression : « cercle carré ».

\subsection{La référence en linguistique}

La référence peut se définir comme «la relation qui va d'une grandeur sémiotique vers une autre non sémiotique (= le référent) relevant, par exemple, du contexte extra-linguistique $»^{60}$. Confrontée aux difficultés d'élucider cette référence, la linguistique contemporaine s'est engagée dans plusieurs voies ${ }^{61}$, mais en outre elle a

\footnotetext{
57. LU XIX/1, p. 85 sq. ; RL II/1, p. 91 sq. Ce problème bien connu est le suivant : les significations essentiellement occasionnelles semblent remettre en cause le principe cardinal de l'unité idéale de toute signification : comment des significations idéales objectives parfaitement fixées pourraient-elles posséder un rapport à des objets individualisés, changeants et liés à la situation subjective du locuteur?

58. Cette question a été précédée par celle des représentations sans objets en 1894 (cf. SOI). Le texte de Twardowski à l'origine des réflexions husserliennes est: Sur la théorie du contenu et de l'objet des représentations (1894).

59. $L U X \mathrm{XIX} / 1$, p. 59 ; RL II/1, p. 61.

60. Greimas, Courtès, DRTL, article « référence ».

${ }^{61}$. Greimas, Courtès (DRTL, article « référent») dégagent quatre orientations possibles. Celle d'Ogden et Richards où un concept médiatise le rapport entre le symbole et l'objet; celle de
} 
parfois choisi de mettre purement et simplement entre parenthèses la référence des signes pour tenter une description de la signification « en immanence » (Greimas, Hjelmslev). Ce choix nous interesse particulièrement parce qu'il implique une prise de distance radicale par rapport aux positions phénoménologiques.

Déjà Saussure, à la suite de l'affirmation du caractère arbitraire du rapport du signe à l'objet, avait suggéré la possibilité de négliger ce rapport dans l'étude de la signification : en etendant à la signification le principe selon lequel «dans la langue il n'existe que des différences », on pouvait envisager de développer une étude de la signification purement en immanence ${ }^{62}$. Avec Hjelmslev, cette possibilité se réalise pour devenir une véritable théorie du langage comme pur système sémiotique. Le langage est un système clos que la théorie domine intégralement en déployant une suite de propositions à partir de définitions de base. Ce principe de clôture a comme présupposé la possibilité d'étudier le langage en mettant entre parenthèses la référence. À tous ses niveaux (texte, phrase, morphème, phonème...) le langage n'est qu'un réseau de relations (de dépendance unilatérale, d'interdépendance, de dépendance sans présupposition) et ses " éléments » de sont que des points d'intersection de ces relations. L'analyse différentielle en immanence ne vaut pas seulement pour le plan de $l^{\prime}$ ' expression " (l'analyse des phonèmes en traits distinctifs) mais aussi pour le plan du «contenu » (du sens) ${ }^{63}$. Chaque plan du langage est structuré en immanence de manière analogue : un « contenu » reçoit une « forme ». Au niveau de l'analyse de la signification (plan du contenu), la «forme du contenu ${ }^{64}$ transforme le sens amorphe (le pur contenu signifiant indéterminé) en substance

\footnotetext{
Jakobson où le référent s'identifie au contexte dans une situation d'énonciation; celle J. Lyons qui pose le référent comme chose en tant qu'objet nommé ou signifié (opposition qui nous semble assez proche de celle de Husserl) ; et enfin leur propre conception, qui évite selon eux les difficultés de mise en relation d'un univers non signifiant et d'un l'univers sémiotique pensé en immanence, puisqu'elle présuppose l'existence d'une sémiotique du monde naturel.

62. Saussure, $C L G$, p. 166. Dans le Cours ce principe domine non seulement l'analyse de la forme phonique mais aussi l'analyse de la signification. (Jakobson refuse cette extension). Les concepts, dit Saussure, «sont purement différentiels, définis non pas positivement par leur contenu, mais négativement par leurs rapports avec les autres termes du système. Leur plus exacte caractéristique est d'être ce que les autres ne sont pas » (CLG, p. 162). On ne saurait se situer plus loin de la thèse husserlienne de l'unité idéale de la signification parfaitement identifiable comme telle et en elle-même.

63. Hjelmslev substitue à la distinction saussurienne entre signifiant et signifié la distinction entre le plan du contenu (du sens) et le plan de l'expression - la langue devient une sémiotique biplane (PTL, p. 78). Cette distinction ne signifie pas indépendance mais au contraire détermination réciproque ou « interdépendance » puisque l'expression et le contenu sont les « fonctifs » (les variables) de la «fonction sémiotique » (PTL, p. 49). Entre les deux plans il y a analogie de structure ; de part et d'autre fonctionne le même type d'articulation en immanence et purement différentielle, mais sur fond d'une distinction de substance fermement maintenue (le son n'est pas le sens).
}

64. PTL, p. 70. 
signifiante articulée, tout de même que sur l'autre plan la forme de l'expression transforme la pure expressivité amorphe (le pur contenu expressif indéterminé) en substance expressive articulée.

Que la forme du contenu relève d'une analyse purement différentielle, deux exemples de Hjelmslev l'illustrent. Le premier compare la manière dont cinq langues (anglais, danois, finnois, français, esquimau) structurent différemment un même contenu de pensée dans cinq énoncés. "Le sens "non-formé" que l'on peut extraire de ces chaines linguistiques prend forme de façon différente dans chaque langue. Chacune d'elle établit ses frontières dans la "masse amorphe de la pensée" en mettant en valeur des facteurs différents dans un ordre différent, place le centre de gravité différemment et donne aux centres de gravité un relief différent $\gg{ }^{65}$ L'exemple de la couleur va dans le même sens. Le français et le gallois, en vertu de leurs formes respectives, découpent différemment la zone de sens touchant au spectre des couleurs ${ }^{66}$.

Greimas, dans une perspective comparable et tout aussi éloignée de la perspective phénoménologique, a de son côté jeté les fondements dans les années soixante d'une sémantique élaborée selon une démarche distributionnelle ${ }^{67}$. Il construit une théorie cohérente à partir de trois concepts fondamentaux - "sémème », "lexème ", « sème » - dans le but d'analyser en immanence le plan du contenu du langage (selon la terminologie de Hjelmslev). Le sémème est le sens particulier d'un mot, il s'analyse en une "figure nucléaire » et des «classèmes » qui sont composés de sèmes, entités minimales purement différentielles et oppositives relevant du plan du contenu (comparables aux phèmes composant les phonèmes au plan de l'expression). Les sèmes fournissent une série de traits distinctifs dont l'organisation spécifique est propre à tel ou tel sémème déterminé. (Le sémème n'est pas une simple liste de sèmes, mais une organisation hypotaxique de sèmes) ${ }^{68}$. Les sémèmes permettent à leur tour de construire les lexèmes par adjonction de formants ${ }^{69}$. Le projet de Greimas est donc bien d'analyser le champ sémantique en immanence et dans une perspective sémiotique, par une analyse distributionnelle des «sèmes » composant les « sémèmes » (eux mêmes exprimés en « lexèmes » $)^{70}$. L'ouvrage Sémantique structurale donne un exemple simplifié $d^{\prime}$ analyse sémique du lexème "spatialité » ${ }^{71}$. Ce

\footnotetext{
65. PTL, p. 70.

66. PTL, p. 70-71.

67. Greimas, Sémantique structurale, Paris, Larousse, 1966, Abrév. SS.

68. ASD, p. 186.

69. Sur les difficultés du concept de lexème, cf. DRTL, article lexème ; Courtès, ASD, p. 189-192.

Le lexème relève de l'usage autant et plus que de la structure sémiotique de la langue.

70. DRTL, articles « sémème », « lexème », « sème ».

71. SS, p. 34-36.
} 
dernier est d'abord analysé en lexèmes plus simples (haut, long, large, vaste, épais), mais - là réside l'originalité de la démarche - ces lexemes plus simples sont reconduits étape par étape à un pur système différentiel de sèmes. Tout le contenu sémantique du lexeme "spatialité " est d'abord réduit à trois oppositions fondamentales de sèmes: dimensionnalité vs non-dimensionnalité, verticalité vs horizontalité, perspectivité vs latéralité. Toutefois, comme dans ce système d'oppositions chaque entité (par exemple la dimensionnalité) se résout elle-même à son tour en un système d'oppositions, puis ce système à son tour en un autre, de proche en proche c'est tout le contenu sémantique du lexème "spatialité » qui se résout en un système complexe de sèmes où chaque entité n'existe qu'en s'opposant à toutes les autres.

Notre étude en appelle une seconde, confrontant les perspectives de la "grammaire pure » husserlienne - qui, au delà du mot, étudie l'agencement des mots en phrases selon des règles syntaxiques relevant d'un a priori matériel (celui de la cohérence sémantique) et les perspectives linguistiques plus récentes - grammaire générative de Chomsky, fondee essentiellement sur des catégories syntagmatiques (syntagme nominal, verbal...), grammaires d'inspiration logicienne (Adjukiewicz, Bar-Hillel) fondęes sur des catégories morphologiques (substantif, adjectif, adverbe...), grammaire actantielle de Greimas. Trois problèmes se posent.

1) La grammaire pure husserlienne est de nature foncièrement sémantique, mais la théorie syntaxique chomskyenne n'intègre (à tous les stades de on évolution) les considérations sémantiques qu'avec les plus grandes difficultés. C'est un ici un point d'opposition sérieux entre la linguistique contemporaine et la phénoménologie.

2) Le second problème touche aux rapports de la grammaire husserlienne avec la logique - donc avec les grammaires logiciennes contemporaines. Chez Husserl, la grammaire comme étude de la morphologie et de la syntaxe des langues naturelles est parallèle à la morphologie et à la syntaxe logiques. Mais elle se distingue de la logique en ceci qu'elle fournit à cette dernière un vêtement expressif qui ne la reflète jamais parfaitement. Dans l'attitude d'élucidation propre au grammairien, le contenu logique n'est pas donné comme tel dans sa pureté idéale à un acte de pensée se réfléchissant luimême dans la parfaite transparence à lui même, mais "vêtu grammaticalement ».

3) L'origine de cet écart entre grammaire et logique, c'est que la grammaire ne rompt pas en 1901 tous les liens avec la psychologie. Les grammaires contemporaines se sont débarrassées de toute perspective psychologique, mais ce n'est pas le cas de la grammaire pure husserlienne en 1901. L'acte expressif envisagé dans sa dimension de facticité ne disparaît pas de l'analyse, puisqu'il est considéré comme 
la réalisation de l'unité idéale de la signification. Il excède donc le domaine des actes purement idéaux où se situent précisément les actes logico-formels, de sorte qu'il représente effectivement pour ces derniers comme un vêtement où ils perdent de leur pureté idéale originaire. Le parallélisme n'est donc jamais parfait entre les formes grammaticales et les formes de la logique pure, parce que les premières, mais non pas les secondes, tout en étant des formes idéales (cf. les analyses de la forme idéale du signifier), sont des formes idéales de certaines expressions factuellement réalisées (utilisées par les individus réels d'une communauté particulière). Les formes de la logique pure sont, elles, totalement déliées de cet ancrage factuel. 